\title{
IMMUNOTHERAPY TREATMENT AGAINST CERVICAL CANCER
}

\author{
Roberto Jiménez-Lima ${ }^{1,2}$, Eder Arango-Bravo ${ }^{2,3}$, Tatiana Galicia-Carmona ${ }^{2,3}$, \\ Leonardo S. Lino-Silva ${ }^{4}$, Guadalupe E. Trejo-Durán ${ }^{5}$, Cristina Alvarado-Silva ${ }^{6}$, \\ Omar H. Castañeda-Renderos ${ }^{7}$, Elva G. Vanoye-Carlo ${ }^{8}$, Celia Flores-de la Torre ${ }^{9}$, \\ Alfonso Dueñas-GonzálezZ ${ }^{2,3 *}$, and Lucely Cetina-PÉrez ${ }^{2,3 *}$
}

\begin{abstract}
${ }^{1}$ Faculty of Medicine, Doctorate Program in Medical, Dental and Health Sciences, Universidad Nacional Autónoma de México (UNAM), Mexico City, Mexico; Departments of ${ }^{2}$ Clinical Research, ${ }^{3}$ Medical Oncology, ${ }^{4}$ Oncologic Pathology, and ${ }^{5}$ Radiotherapy, Instituto Nacional de Cancerología (INCan), Mexico City, Mexico ${ }^{6}$ Department of Medical Oncology, Hospital Juárez de México, Mexico City, Mexico; ${ }^{7}$ Department of Medical Oncology, Hospital Universitario de Saltillo, Coah., Mexico; ${ }^{8}$ Department of Gynecologic Oncology, Hospital General Agustín O’Horan Mérida, Yuc., Mexico; and ${ }^{9}$ Department of Medical Oncology, Centro Estatal de Oncología Campeche INDESALUD, Camp., Mexico
\end{abstract}

\begin{abstract}
Cervical cancer (CC) is one of the most common gynecological tumors and an important health problem, especially in developing countries. The vast majority of patients in early stages are cured of the disease with surgical treatment and with concomitant chemoradiotherapy in locally advanced stages. However, in patients with recurrent, persistent, or metastatic cervical CC, the effectiveness of treatment is limited, except for the combination of chemotherapy based on platinum doublets plus bevacizumab, the treatment that has achieved the best results to date. Programmed cell death-1/PD ligand-1 (PD-1/PD-L1) inhibitors could be a novel and cutting-edge therapeutic option to improve clinical outcomes in this group of patients. Thus far, there are a few Phase I/II clinical trials that have assessed the usefulness of pembrolizumab and nivolumab in this group of patients; these include the KEYNOTE 028, KEYNOTE 158, and CHECKMATE 358 trials, in which clinical benefit has been proven with PD-1/PD-L1 inhibitors in recurrent, persistent, or metastatic CC, as second-line treatment. There are also some ongoing trials that could provide further evidence on the PD-1/PD-L1 pathway as a therapeutic target in CC. In this review, we will focus on the usefulness of these PD-1/PDL1 inhibitors in CC, as well as on trials that are still in the recruitment phase, to confirm their effectiveness in this clinical setting. (REV INVEST CLIN. 2020;72(4):231-8)
\end{abstract}

Key words: Immunotherapy. Cytotoxic T-lymphocyte-associated protein 4. Programmed cell death-1/Programmed cell death ligand-1. Checkpoint. Monoclonal antibodies.

*Corresponding author:

Lucely Cetina-Pérez/Alfonso Dueñas-Gonzalez

E-mail: lucelycetina.incan@gmail.com
Received for publication: 20-02-2020

Approved for publication: 20-04-2020

DOI: $10.24875 /$ RIC.20000060

0034-8376 / (c) 2020 Revista de Investigación Clínica. Published by Permanyer. This is an open access article under the CC BY-NC-ND license (http://creativecommons.org/licenses/by-nc-nd/4.0/). 


\section{INTRODUCTION}

Cervical cancer (CC) remains an important public health problem in Mexico ${ }^{1}$ since it ranks second in terms of morbidity and is one of the leading causes of death in women of reproductive age ${ }^{2}$. More than $80 \%$ of cases of CC are diagnosed at locally advanced stages (IB2-IVA) and metastatic IVB stage. In early stages, overall survival (OS) can reach approximately $75-85 \%$ with effective treatments such as surgery or concomitant chemoradiotherapy ${ }^{3}$. However, 5-year OS for recurrent, persistent, or metastatic CC is only a mere $15 \%$. This poor prognosis is mainly due to limited treatment options ${ }^{4}$. Most of these patients can only be treated with palliative chemotherapy (CT), based on cisplatin doublets ${ }^{5}$. In 2014, based on the results of the Gynecologic Oncology Group (GOG) 240 trial, it was concluded that the addition of bevacizumab to $\mathrm{CT}$ with doublets improved progressionfree survival (PFS) from 6 months to 8.2 months and OS from 13 to 17 months in recurrent, persistent or metastatic CC, and is currently considered the standard treatment in these clinical stages ${ }^{6}$. Nevertheless, in patients who progress during first-line treatment, the lack of an effective second-line treatment remains the main cause of the high mortality rate.

Several approaches have been tested in the last decade aimed to improve OS in different neoplasms. In this regard, the introduction of immune checkpoint inhibitors, especially programmed cell death-1/programmed cell death ligand-1 (PD-1/PD-L1) inhibitors ${ }^{7}$, has achieved favorable efficacy results for the treatment of multiple solid tumors such as lung and kidney cancer, among others ${ }^{8}$. Furthermore, immunotherapy is currently being tested for CC in different clinical trials to address its effectiveness and tolerability in second-line therapies.

In this review, we present the current data aimed to targeting PD-1, PD-L1, and CTL-4 pathways through different monoclonal antibodies to disrupt immunologic tolerance as a promising targeted therapy for $\mathrm{CC}^{9}$.

Patient, intervention, control, and outcome acronym question was used to detect articles, including checkpoint inhibitors, immunotherapy treatment, PD1, PD-L1, CTL-4, and CC. The search was conducted using the MEDLINE and the PubMed database browsers combining the following terms: "CC," cancer," "cytotoxic T-lymphocyte-associated protein 4 (CTLA-4)," "PD-1," "PD-L1," "immunocheckpoint," "monoclonal antibodies," "therapeutic target," and "immunotherapy." Only articles published in the past 15 years and in English were included to ensure the most updated collection of scientific evidence.

All authors performed the revision and extraction of data from the original manuscripts. The first author was contacted to obtain those articles, in which the final version was not available online, and thus, no information was lost. Finally, articles were revised and the information processed according to the GRADE system; evidence was classified, and recommendations were then posed according to the strength of the evidence.

\section{IMMUNE CHECKPOINT INHIBITORS}

Numerous immunomodulatory therapies are being investigated in various clinical trials with different potential targets, including PD-1/PD-L1, CTLA-4, Tim-3, inducible costimulator (ICOS), 4-1BB, and OX-40. Among these new targets, ICOS, 4-1BB, and OX-40 are costimulatory receptors ${ }^{10-12}$, while PD-1/PD-L1, CTLA-4, and Tim-3 are T-cell immune downregulators ${ }^{13-15}$. At present, only CTLA-4 inhibitors and PD-1/PD-L1 inhibitors have been approved by the Food and Drug Administration (FDA). CTLA-4 is a part of the CD80 (B7-1) and CD86 (B7-2) costimulatory molecules that are expressed on the surface of antigen-presenting cells (APCs) ${ }^{16}$, while PD-L1 is expressed in a wide variety of cell types, including tumor-associated fibroblasts, tumor cells (TCS), and APCs, among others ${ }^{17}$. Thus, CTLA-4 inhibits T-cell activation in secondary lymphoid organs, but PD-1/ PD-L1 mainly regulates T-cell function in peripheral tissues and in the tumor microenvironment. Therefore, PD-1/PD-L1 signaling is more tumor specific than CTLA-4 signaling, and PD-1/PD-L1 inhibitors may cause less damage to healthy tissue ${ }^{18}$.

The CTLA-4 and PD-1/PD-L1 pathways regulate immune system activation at different activation points and through different effector cells. CTLA-4 inhibits the $T$-cell response based on antigen-binding affinity. CTLA-4 is stored in intracellular vesicles, and when compensation of the response becomes necessary, it 
is translocated to the cell surface. In contrast, PD-1/ PD-L1 is expressed in peripheral tissues to compensate for immune responses within the context of inflammation, which limits injury to healthy tissue ${ }^{19}$.

Based on the above mechanism, ipilimumab (monoclonal anti-CTLA-4 antibody), the first immune checkpoint inhibitor approved for melanoma, had little clinical benefit until the appearance of pembrolizum$a b$, and the combination of both drugs further improved treatment efficacy in malignant melanoma ${ }^{20}$. To date, another available monoclonal anti-CTLA-4 antibody is tremelimumab; however, it has yet to be approved in the treatment of any type of cancer. In addition, the PD-1-targeted monoclonal antibodies pembrolizumab, nivolumab, and cemiplimab, as well as those targeting the PD-L1 ligand, atezolizumab, durvalumab, and avelumab, have shown clinical advantages in malignant melanoma, advanced non-small cell lung cancer (NSCLC), urothelial cancer, and other tumors ${ }^{18}$. Furthermore, extensive research has been conducted on gynecological tumors, such as ovarian cancer and endometrial cancer. Furthermore, clinical trials on CC are being conducted and a few initial results have been obtained.

\section{RATIONALE FOR PD-1/PD-L1 INHIBITORS IN CERVICAL CANCER}

The development of cervical intraepithelial neoplasia (CIN) and CC is related to persistent human papillomavirus (HPV) infection. The cellular immune response is responsible for the elimination of the virus and infected cells and is one of the factors that influence the persistence of infection. Among the molecules that regulate the immune response are PD-1 and PD-L1. The expression of these molecules is increased in patients with high-risk HPV infection and correlates with CIN grade. Activation of the PD-1/ PD-L1 pathway correlates with deficiencies in the cellular immune response against high-risk HPV infection and is, therefore, implicated in the immunopathogenesis of cervical lesions ${ }^{21}$.

PD-1/PD-L1 immune control pathways are one of the best-known immune evasion mechanisms of cancer cells and, therefore, inhibit the immune response in several types of solid tumors, including CC. Briefly, PD-L1 is expressed on the surface of cervical TC,
APCs, and tumor-infiltrating lymphocytes (TILs), while PD-1-positive cells have been mainly identified as $T$ cells in the stroma of cervical tumors. PD-1 expression in the tumor stroma of CC was reported by Meng et al. to be present in $60.82 \%(59 / 97)$ of patients $^{22}$, while another study showed PD-1 expression in $46.97 \%(31 / 66)$ of patients ${ }^{23}$.

So far, numerous studies have investigated PD-L1 expression in CC, reporting that PD-L1 expression ranges between $34.4 \%$ and $96 \%$ in cervical carcinoma tissues, while PD-L1 expression is rarely present or identified in histologically normal cervical tissues ${ }^{24}$. Reddy et al. showed that PD-L1 expression was positive in $34.4 \%$ of patients with cervical carcinoma samples, and when performing a sub-analysis by histologic subtypes, the expression varied by histologic type: $37.8 \%$ in squamous cell, $28.6 \%$ in adenosquamous carcinomas, and $16.7 \%$ in adenocarcinoma ${ }^{25}$. In addition, PD-L1 can also be expressed in TIL that plays a role in antitumor response inhibition. One study in cervical squamous cell carcinoma samples found that PD-L1 expression rate in cancer cells and TIL was $59.1 \%$ and $47.0 \%$, respectively ${ }^{23}$. Taken together, these data suggest that both PD-L1 and PD-1 are widely expressed in CC TC and in the stroma, providing possible therapeutic targets for PD-1/PD-L1 inhibitors.

Notoriously, persistent HPV infection is involved in the pathogenesis of CC and is related to its prognosis. Several groups have questioned whether HPV infection might affect PD-L1 expression in CC and have found that the association of HPV infection correlated positively with increased PD-L1 expression ${ }^{26}$.

Considerable effort has been made to analyze the mechanism underlying the association between HPV status and PD-L1 expression in solid HPV-related tumors, mainly head-and-neck squamous cell tumors and CC. In CC, HPV-induced somatic mutations and a large number of neoantigens have been reported to play a crucial role in the tumor microenvironment and could lead to notable alterations between immune system checkpoint regulator-related genes such as CTLA-4, PD-1, and PD-L127. Specifically, PD-L1 showed a positive correlation with ENO1, PRDM1, OVOL1, and MNT, all of which are key regulators related to HPV16 and its proteins $\mathrm{E} 6$ and $\mathrm{E} 7^{28}$. Of note, a Phase II single-arm study investigated durvalumab 
in patients with recurrent/metastatic head-and-neck squamous cell carcinoma (HNSCC) $(n=112)$ and found that HPV-positive patients had a higher response rate and better survival than HPV-negative subjects ${ }^{27}$. However, in CC, the association of HPV status and PD-1/PD-L1 inhibitor efficacy is still uncertain due to the lack of available data.

\section{CLINICAL INVESTIGATION OF DIFFERENT PD-1/PD-L1 INHIBITOR MONOCLONAL ANTIBODIES IN CERVICAL CANCER}

\section{Pembrolizumab}

Pembrolizumab is an immunoglobulin G4 (IgG4)-type humanized monoclonal antibody that targets the PD-1 receptor found on the cell surface. It is a checkpoint inhibitor with immune potential and antineoplastic activity. Pembrolizumab binds to the PD-1 receptor and blocks its interaction with the PD-L1 and PD-L2 ligands, which are expressed in TC and other cells in the tumor microenvironment, and enhances $\mathrm{T}$ cells immune response, including the anti-tumor response ${ }^{21}$.

The KEYNOTE-028 Phase $1 \mathrm{~b}$ trial (ClinicalTrials.gov NCT02054806) ${ }^{29}$ is investigating the safety and efficacy of the checkpoint inhibitor pembrolizumab in PD-L1-positive advanced solid tumors. The study included 24 patients with advanced CC with positive PD-L1 expression who were not candidates or had failed standard therapy. Previous radiation therapy had been received by $92 \%$ of patients and $63 \%$ had received two or more lines of $\mathrm{CT}$, including bevacizumab. Patients were treated with $10 \mathrm{mg} / \mathrm{kg}$ of pembrolizumab every 2 weeks for 24 months or until evidence of disease progression appeared, unacceptable toxicity, or by the investigator's decision. Response was assessed every 8 weeks during the first 6 months and then every 12 weeks, with an overall response rate (ORR) according to Response Evaluation Criteria in Solid Tumors (RECIST) of 17\% (95\% confidence interval [Cl]: 5-37); four patients had a partial response (PR) with a mean response duration of 5.4 months (4.1-7.5 months), and three patients showed stable disease (SD). No complete response (CR) was observed, and two-thirds of the patients had a progressive disease. OS was 11 months, and at 6 months, $67 \%$ of the patients were alive. In terms of toxicity, five patients developed Grade 3 adverse events (AEs)
(NCl-CTCAE 3.0), and no Grade 4 AEs were observed $^{29}$.

The results of the KEYNOTE-028 trial in patients with CC have allowed pembrolizumab to be assessed in a Phase 2 open-label study, the KEYNOTE-158 trial (ClinicalTrials.gov - NCT02628067) $^{30}$. This study is evaluating the response of several solid tumors that have progressed with standard therapy and that are candidates for treatment with pembrolizumab. In the CC cohort, 98 patients with recurrent or metastatic CC were included. With a median follow-up time of 11.7 months, ORR in 77 patients was $14.3 \%$ ( $95 \% \mathrm{Cl}$ : 7.4-24.1\%), including $2.6 \%$ of patients with CR and $11.7 \%$ with PR, while no response was observed in patients without PD-L1 expression in TCs. The most common serious adverse reactions included anemia (7\%), fistula (4.1\%), hemorrhage (4.1\%), and infection (4.1\%). Based on the results of KEYNOTE 158 , the FDA approved pembrolizumab on June 12, 2018, for advanced CC with disease progression during or after CT30. Furthermore, based on these results, KEYNOTE 826 recently initiated the recruitment phase, and its aim is to assess the efficacy and safety of first-line treatment with pembrolizumab (MK-3475) plus $C T$ versus placebo plus $C T$ in women with persistent, recurrent, or metastatic CC (MK-3475-826/ KEYNOTE-826).

\section{Nivolumab}

Nivolumab is a PD-1 immune checkpoint inhibitor designed to enhance the immune system response and restore the antitumor immune response. It is an IgG4type human monoclonal antibody that enhances Tcell responses, including the antitumor response, by binding to the PD-1 receptor and blocking its interaction with PD-L1 and PD-L2 ${ }^{31}$.

At present, treatment with nivolumab is approved for non-operable and/or metastatic melanoma, as well as second-line treatment of squamous cell lung cancer and clear cell renal cell carcinoma ${ }^{31}$.

The CHECKMATE 141 study is a Phase III clinical trial that included patients with head-and-neck neoplasms that were randomized to receive nivolumab $(3 \mathrm{mg} / \mathrm{kg}$ every 2 weeks) or standard CT. Mean OS was longer in the experimental group (7.5 vs. 5.1 months; hazard 
ratio [HR]: $0.70 ; 97.73 \% \mathrm{Cl}: 0.51-0.96 ; \mathrm{p}=0.001)$, with no impact on PDL-1 expression ( $>1 \%$ vs. $<1 \%)^{32}$.

The open-label Phase $1 / 2$ study, CHECKMATE 358 (ClinicalTrials.gov - NCT02488759) $^{33}$, is assessing treatment with $240 \mathrm{mg}$ of nivolumab every 2 weeks until progression or unacceptable toxicity in patients with HPV-associated tumors (cervix, vagina, and vulva). Preliminary results from 24 patients showed an ORR of $20.8 \%$ (95\% Cl: 7.1-42.2) and a disease control rate of $70.8 \%$ defined as PR, CR, or SD. PFS was 5.5 months ( $95 \% \mathrm{Cl}: 3.5$ - not reached) and mean OS had not been achieved. Responses were only observed in patients with CC (19 out of 24), with an ORR of $26.3 \%$ (95\% Cl: 9.1-51.2). Response mean duration had not been reached after 6 months of follow-up. The response was independent of PD-L1 or HPV status or of the number of previous therapies. Treatment-related AEs were reported in $70.8 \%$ of patients, and $12.5 \%$ of AEs were Grades 3-4, including hyponatremia, syncope, diarrhea, and hepatocellular injury ${ }^{33}$. Thus, considering the previously discussed pembrolizumab and nivolumab studies, in both cases, the drugs showed promising antitumor effects and, in addition, they had an adequate toxicity profile in patients with recurrent or metastatic CC. However, due to the limited follow-up period, PFS and OS have yet to be reported because the data are initial ${ }^{29-33}$.

\section{Atezolizumab}

Atezolizumab is a humanized monoclonal antibody that binds to the PD-L1 ligand, which is expressed in both TCs and immune cells (ICS) infiltrating the tumor, and blocks its binding with PD-1 and B7-1 (CD80). This restores T-cell activity, including the antitumor immune response, by preventing the inhibition of the immune response mediated by PD-L1/PD-1 binding, without inducing antibody-dependent cell-mediated cytotoxicity ${ }^{19,34}$.

There are numerous Phase I/II trials, in which the usefulness of this molecule has been assessed in several neoplasms such as NSCSC, including BIRCH, FIR, POPLAR, and OAK, the latter being a Phase III, and IMVIGOR 210 a Phase II study on bladder cancer. In all these studies, atezolizumab was administered at a fixed dose of $1200 \mathrm{mg}$ IV every 21 days $^{35}$. Thus, the OAK study ${ }^{36}$, in a Phase III clinical trial randomized
850 patients diagnosed with Stage IIIB or IV NSCLC with progression to 1 or 2 previous lines of treatment and who met eligibility criteria, received atezolizumab $1200 \mathrm{mg}$ versus docetaxel $75 \mathrm{mg} / \mathrm{m}^{2}$ every 3 weeks. This study showed positive results in its primary endpoint: OS was superior with atezolizumab in comparison with docetaxel (13.8 months vs. 9.6 months; HR: 0.73 ; $95 \% \mathrm{Cl}: 0.62-0.87 ; \mathrm{p}=0.0003$ ). Atezolizumab was also superior to docetaxel in the PD-L1positive population (15.7 months vs. 10.3 months; HR: $0.74 ; 95 \% \mathrm{Cl}: 0.58-0.93 ; \mathrm{p}=0.0102$ ). The largest benefit was observed in patients with the highest PDL1 expression (TC3 or IC3), with a mean OS of 20.5 months with atezolizumab versus 8.9 months with docetaxe ${ }^{36}$. Based on efficacy and safety in the OAK and POPLAR studies (Phase II of atezolizumab in NSCLC), the use of atezolizumab was approved for patients with NSCLC after progression to platinumbased CT, regardless of the PD-L1 status ${ }^{36}$.

The IMVIGOR 210 study is a clinical trial (Phase II), in which the usefulness of atezolizumab was assessed in patients with metastatic bladder cancer. This trial had two analysis cohorts. The first cohort included patients who were not candidates for platinum therapy, with a benefit in objective response rates of $23 \%$ and a median OS of 15.9 months regardless of the degree of PDL-1 expression, with adequate treatment tolerance and manageable $\mathrm{AEs}^{35}$.

IMVIGOR 210 is Cohort 2 and included patients with bladder cancer and progression to previous platinumbased therapies. Overall, the objective response rate was $48 \%$; however, it was higher in patients with PDL1 expression (IC 2/3). Similarly, OS was 11.4 months versus 6.5 months when comparing PDL-1 expression (IC $2 / 3$ vs. IC 0 ), with the benefit favoring the use of atezolizumab ${ }^{35}$.

Several studies are currently underway, evaluating treatment with atezolizumab alone or in combination in patients with recurrent, persistent, or metastatic CC.

The PROLOG study (ClinicalTrials.gov - NCT02914470) is a Phase I trial that is assessing the CT effective dose of carboplatin plus cyclophosphamide in combination with atezolizumab in several types of gynecological cancer, including CC. The primary objective of the study is toxicity assessment, and the secondary is ORR according to RECIST criteria. 
One more trial in which the combination of atezolizumab with bevacizumab is being analyzed is a Phase II study (ClinicalTrials.gov - NCT02921269). This study will assess the effectiveness of the combination in the reduction of TCs and their interference with their ability to grow and spread. The primary endpoint is ORR, defined according to RECIST criteria, with secondary endpoints being PFS, OS, the incidence of AEs, PD-L1 expression in the tumor, and ICs measured by semi-quantitative immunohistochemistry, as well as the determination of intra-tumor T-cell clones and peripheral cells measured by TCR sequencing. One more Phase II trial will establish the safety and efficacy of atezolizumab in patients with recurrent $\mathrm{CC}$ as second-line treatment. The study will consist of three treatment arms: atezolizumab monotherapy, atezolizumab in combination with doxorubicin, and doxorubicin monotherapy. The primary endpoint of the study is PFS and the secondary is OS, in all three treatment arms.

Finally, a Phase III study is soon to be conducted (ClinicalTrials.gov - NCT03556839), to determine the efficacy of combining atezolizumab plus bevacizumab with the current standard of treatment (cisplatin plus paclitaxel) in patients with Stage IVB persistent or recurrent carcinoma of the cervix. The primary endpoint is OS, and secondary endpoints are PFS, ORR, response duration, the incidence of treatment-related AEs, time to the next therapy, PFS 2, patient-reported results regarding functionality and quality of life, atezolizumab pharmacokinetics, and the incidence of antitherapeutic antibodies.

\section{Durvalumab}

Durvalumab is a human monoclonal antibody that blocks the PD-L1 ligand. Durvalumab acts as a selective antibody against PD-L1 and prevents its interaction with the PD-1 receptor, which leads to T-cell normal antitumor activity ${ }^{37}$.

At present, no results were found in the effectiveness of durvalumab in CC, and the vast majority of available studies are still in the recruitment phase. Nevertheless, some results about this molecule in other neoplasms were found, such as the use of durvalum$\mathrm{ab}$ in patients with squamous cell carcinomas of the head and neck ${ }^{38}$. Thus, in a Phase II study that assessed the use of durvalumab in patients with headand-neck tumors (squamous cell carcinoma) with progression to a previous platinum-based first line, a response rate of $16.2 \%$ was reported, and $29.4 \%$ in patients with tumors with HPV versus $10.8 \%$ in those without the presence of the virus. With regard to survival curves, the median was 7.1 months, and when the analysis was performed for those who were $\mathrm{HPV}+$, it was 10.2 months versus 5.0 months in HPVsubjects ${ }^{27}$.

The use of durvalumab in combination with tremelimumab in solid tumors is being assessed in a Phase I study (ClinicalTrials.gov - NCT01975831). The primary objective of the study is the number of AEs, and secondary objectives include determining the pharmacokinetics of the durvalumab plus tremelimumab combination, the number of subjects who develop antibodies against the molecule, tumor response, PFS, and $O S$.

Two Phase Istudies (ClinicalTrials.gov-NCT03452332 and NCT03277482) will evaluate the safety of using durvalumab and tremelimumab in combination with stereotactic body radiation therapy and radiotherapy, respectively, in patients with recurrent or metastatic gynecological cancer. The primary objective of the first study is to determine the dose-limiting toxicities based on AEs, and the secondary objectives are clinical and objective response rates, PFS, OS, and time to the next treatment. The second study has as its primary objective to determine the maximum tolerated dose, and secondary objectives include ORR, response rates and local control, response duration, PFS, or OS.

There are two more studies, Phases I and II, still in the recruitment phase, that will assess the use of durvalumab in combination with the DNA plasmid-encoding interleukin 12/HPV DNA plasmid therapeutic vaccine (INO-3112), in patients with HPV-associated cancer (ClinicalTrials.gov - NCT03439085 and NCT03162224). Both studies will evaluate the efficacy and safety of the combination. In addition, a Phase III clinical trial (CALLA), in the setting of locally advanced CC, is going to assess the combination of durvalumab with concomitant chemoradiotherapy with cisplatin plus maintenance for 2 years. The results are expected in the coming years. 
Durvalumab will also be assessed in combination with vigil in a Phase II study (ClinicalTrials.gov NCT02725489) and in combination with tremelimumab plus metronomic vinorelbine in a Phase I/II study (ClinicalTrials.gov - NCT03518606).

\section{Cemiplimab}

Cemiplimab is a human recombinant monoclonal antibody of the IgG4 subtype that binds to the PD-1 receptor and blocks the binding of the PD-L1 and PD-L2 ligands. This eliminates immune response inhibition by PD-1 and has been associated with tumor reduction ${ }^{39}$.

The GOG3016 study is the first Phase III trial that will evaluate the use of immunotherapy to treat $\mathrm{CC}^{40}$. Cemiplimab assessment went directly to Phase III, after the results obtained in the Phases I and II studies ${ }^{41}$. The Phase I trial assessed the drug in patients with advanced, locally advanced malignant tumors or with squamous cell carcinoma of the skin, while the Phase II study was conducted in patients with metastatic disease. Both studies treated patients with $3 \mathrm{mg} / \mathrm{kg}$ of cemiplimab every 2 weeks. The Phase I study reported a response to cemiplimab in $50 \%$ of patients (95\% Cl: 30-79). Of the three patients with CC included in the Phase I study, two had a lasting response. Similarly, the results of the Phase II study showed that $47 \%$ of patients ( $95 \% \mathrm{Cl}: 34-61$ ) had a response, with a mean follow-up of 7.9 months. Of the 28 patients who responded, its duration exceeded 6 months in $57 \%$. The AEs that occurred in more than $15 \%$ of patients were diarrhea, fatigue, nausea, constipation, and rash, with $7 \%$ of patients discontinuing the treatment due to $\mathrm{AEs}^{41}$.

GOG 3016 (ClinicalTrials.gov - NCT03257267) 40 is a Phase III trial that will assess the efficacy and safety of cemiplimab versus $C T$, in patients $\geq 18$ years of age with recurrent or metastatic platinum-resistant CC that progressed within 6 months of the last dose of platinum-based CT. Patients will receive cemiplimab every 3 weeks or CT (pemetrexed, topotecan, irinotecan, gemcitabine, or vinorelbine). The primary endpoint will be OS, and secondary endpoints will include OS analysis, PFS, and the frequency and severity of AEs. The efficacy analyses will be stratified according to factors such as the previous use of bevacizumab, histology, and ECOG performance. The duration of the recruitment period of the study and follow-up will be 20 and 12 months, respectively, and therefore, it is estimated that 436 patients will have to be included, among which 330 deaths are expected, to achieve a power of $90 \%$ to detect a reduction in the risk of death of at least $20 \%$.

\section{CONCLUSIONS}

Immunotherapy with PD-1 and PD-L1 blockade is in an investigation phase. Based on current evidence, and considering that there are promising results with which the benefits outweigh the deleterious effects, their use should be considered in the context of clinical research and in patients who have progressed to different lines of treatment and there are no more therapies to be offered with well demonstrated clinical effectiveness.

\section{RECOMMENDATIONS}

The use of pembrolizumab is recommended in advanced, recurrent, and persistent CC with progression to at least one previous lines of treatment since it increases objective response rates and median survival, with good tolerance. Quality of evidence: (GRADE) low. Strength of recommendation: weak in favor of its use.

The use of atezolizumab, nivolumab, durvalumab, and cemiplimab is not recommended until the publication of the Phase III studies that are currently being conducted. Quality of evidence: (GRADE) very low. Strength of recommendation: weak against their use.

\section{REFERENCES}

1. Goodman A. HPV testing as a screen for cervical cancer. BMJ. 2015;350:2372.

2. Sahasrabuddhe VV, Parham GP, Mwanahamuntu MH, Vermund $\mathrm{SH}$. Cervical cancer prevention in low-and middle-income countries: feasible, affordable, essential. Cancer Prevent Res. 2012; 5:11-7.

3. Chen J, Gu W, Yang L, Chen C, Shao R, Xu K, et al. Nanotechnology in the management of cervical cancer. Rev Med Virol. 2015;25 Suppl 1:72-83.

4. Guitarte C, Alagkiozidis I, Mize B, Stevens E, Salame G, Lee YC. Glassy cell carcinoma of the cervix: a systematic review and meta-analysis. Gynecol Oncol. 2014;133:186-91.

5. Monk BJ, Sill MW, Mcmeekin DS, Cohn DE, Ramondetta LM, Boardman $\mathrm{CH}$, et al. Phase III trial of four cisplatin-containing 
doublet combinations in stage IVB, recurrent, or persistent cervical carcinoma: a gynecologic oncology. J Clin Oncol. 2009; 27:4649-55.

6. Tewari KS, Sill MW, Long HJ 3rd, Penson RT, Huang H, Ramondetta LM, et al. Improved survival with bevacizumab in advanced cervical cancer. N Engl J Med. 2014;370:734-43.

7. Constantinidou A, Alifieris C, Trafalis DT. Targeting programmed cell death-1 (PD-1) and ligand (PD-L1): a new era in cancer active immunotherapy. Pharmacol Ther. 2019;194:84-106.

8. Gettinger S, Horn L, Jackman D, Spigel D, Antonia S, Hellmann $M$, et al. Five-year follow-up of nivolumab in previously treated advanced nonsmall-cell lung cancer: results from the CA209003 study. J Clin Oncol. 2018;36:1675-84

9. Borcoman E, Le Tourneau C. Pembrolizumab in cervical cancer: latest evidence and clinical usefulness. Ther Adv Med Oncol. 2017;9:431-9

10. Amatore F, Gorvel L, Olive D. Inducible co-stimulator (ICOS) as a potential therapeutic target for anti-cancer therapy. Expert Opin Ther Targets. 2018;22:343-51.

11. Compte M, Harwood SL, Muñoz IG, Navarro R, Zonca M, PerezChacon $\mathrm{G}$, et al. A tumor-targeted trimeric 4-1BB-agonistic antibody induces potent anti-tumor immunity without systemic toxicity. Nat Commun. 2018;9:4809.

12. Polesso F, Weinberg A, Moran AE. Late stage tumor regression after PD-L1 blockade with a concurrent OX40 agonist. Cancer Immunol Res. 2019;7:269-81.

13. Raedler LA. Opdivo (nivolumab): second PD-1 inhibitor receives FDA approval for unresectable or metastatic melanoma. Am Health Drug Benefits. 2015;8:180-3.

14. Lheureux S, Butler MO, Clarke B, Cristea MC, Martin LP, Tonkin K, et al. Association of ipilimumab with safety and antitumor activity in women with metastatic or recurrent human papillomavirusrelated cervical carcinoma. JAMA Oncol. 2018;4:e173776.

15. Gorris MA, Halilovic A, Rabold K. Eight-color multiplex immunohistochemistry for simultaneous detection of multiple immune checkpoint molecules within the tumor microenvironment. J Immunol. 2018;200:347-54

16. Fife BT, Bluestone JA. Control of peripheral T-cell tolerance and autoimmunity via the CTLA-4 and PD-1 pathways. Immunol Rev. 2008;224:166-82

17. Boussiotis VA. Molecular and biochemical aspects of the PD-1 checkpoint pathway. N Eng| J Med. 2016:375:1767-78.

18. Liu Y, Wu L, Tong R, Yang F, Yin L, Li M, et al. PD-1/PD-L1 inhibitors in cervical cancer. Front Pharmacol. 2019;10:65.

19. Pardoll DM. The blockade of immune checkpoints in cancer immunotherapy. Nat Rev Cancer. 2012;12:252-64.

20. Wang J, Chmielowski B, Pellissier J, Xu R, Stevinson K, Liu FX. Cost-effectiveness of pembrolizumab versus ipilimumab in ipilimumab-naive patients with advanced melanoma in the United States. J Manage Care Spec Pharm. 2017;23:184-94.

21. Yang W, Song Y, Lu YL, Sun JZ, Wang HW. Increased expression of programmed death (PD)-1 and its ligand PD-L1 correlates with impaired cell-mediated immunity in high-risk human papillomavirus-related cervical intraepithelial neoplasia. Immunology. 2013;139:513-22.

22. sssMeng $Y$, Liang $H, H u$ J, Liu $S$, Hao $X$, Wong $M S$, et al. PDL1 Expression correlates with tumor infiltrating lymphocytes and response to neoadjuvant chemotherapy in cervical cancer. J Cancer. 2018;9:2938-45.

23. Feng $Y C$, Ji WL, Yue N, Huang YC, Ma XM. The relationship between the PD-1/PD-L1 pathway and DNA mismatch repair in cervical cancer and its clinical significance. Cancer Manage Res. 2018:10:105-13.

24. Enwere EK, Kornaga EN, Dean M, Koulis TA, Phan T, Kalantarian $M$, et al. Expression of PD-L1 and presence of CD8-positive T cells in pre-treatment specimens of locally advanced cervical cancer. Mod Pathol. 2017;30:577-86.
25. Reddy OL, Shintaku PI, Moatamed NA. Programmed death-ligand 1 (PD-L1) is expressed in a significant number of the uterine cervical carcinomas. Diagn Pathol. 2017;12:45

26. Mezache L, Paniccia B, Nyinawabera A, Nuovo GJ. Enhanced expression of PD L1 in cervical intraepithelial neoplasia and cervical cancers. Mod Pathol. 2015;28:1594-602.

27. Zandberg DP, Algazi AP, Jimeno A, Good JS, Fayette J, Bouganim $\mathrm{N}$, et al. Durvalumab for recurrent or metastatic head and neck squamous cell carcinoma: results from a single-arm, phase II study in patients with $\geq 25 \%$ tumour cell PD-L1 expression who have progressed on platinum-based chemotherapy. Eur J Cancer. 2019;107:142-52.

28. Qin Y, Ekmekcioglu S, Forget MA, Szekvolgyi L, Hwu P, Grimm $E A$, et al. Cervical cancer neoantigen landscape and immune activity is associated with human papillomavirus master regulators. Front Immunol. 2017;8:689

29. Frenel JS, Le Tourneau C, O'Neil B, Ott PA, Piha-Paul SA, GomezRoca $C$, et al. Safety and efficacy of pembrolizumab in advanced programmed death ligand 1-positive cervical cancer: results from the phase Ib KEYNOTE-028 trial. J Clin Oncol. 2017; 35:4035-41.

30. Schellens JH, Marabelle A, Zeigenfuss S, Ding J, Pruitt SK, Chung HC. Pembrolizumab for previously treated advanced cervical squamous cell cancer: preliminary results from the phase 2 KEYNOTE-158 study. J Clin Oncol. 2017;35 Suppl 15:5514.

31. Minion LE, Tewari KS. Cervical cancer-state of the science: from angiogenesis blockade to checkpoint inhibition. Gynecol Oncol. 2018:148:609-21.

32. Haddad R, Blumenschein G Jr., Fayette J, Guigay J, Colevas AD, Licitra $L$, et al. Treatment beyond progression with nivolumab in patients with recurrent or metastatic $(R / M)$ squamous cell carcinoma of the head and neck (SCCHN) in the phase 3 checkmate 141 study: a biomarker analysis and updated clinical outcomes. Annals Oncol. 2017:28 Suppl 5:v372-94.

33. Hollebecque A, Meyer T, Moore KN, Machiels JP, De Greve J López-Picazo JM, et al. An open-label, multicohort, phase I/II study of nivolumab in patients with virus-associated tumors (CheckMate 358): efficacy and safety in recurrent or metastatic (R/M) cervical, vaginal, and vulvar cancers. J Clin Oncol. 2017;35 Suppl 15:5504.

34. Drug Bank. Atezolizumab. Available from: https://www.drugbank.ca/drugs/DB11595.

35. Shaha NJ, Kellya WJ, Liu SV, Choquetteb K, Spira A. Product review on the anti-PD-L1 antibody atezolizumab. Hum Vaccin Immunother. 2018;14:269-76.

36. Rittmeyer A, Barlesi F, Waterkamp D, Park K, Ciardiello F, von Pawel J, et al. Atezolizumab versus docetaxel in patients with previously treated non-small-cell lung cancer (OAK): a phase 3 open-label, multicentre randomised controlled trial. Lancet. 2017;389:255-65

37. Drug Bank. Durvalumab. Available from: https://www.drugbank. ca/drugs/DB11714.

38. Alfieri S, Cavalieri S, Licitra L. Immunotherapy for recurrent/ metastatic head and neck cancer. Curr Opin Otolaryngol Head Neck Surg. 2018:26:152-6

39. Markham A, Duggan S. Cemiplimab: first global approval. Drugs. 2018;78:1841-6

40. Tewari KS, Vergote I, Oaknin A, Alvarez E, Chase DM, Gaillard S et al. GOG 3016/ENGOT-cx9: an open-label, multinational, randomized, phase 3 trial of cemiplimab, an anti-PD-1, versus investigator's choice (IC) chemotherapy in $\geq 2$ line recurrent or metastatic cervical cancer. J Clin Oncol. 2018;36 Suppl 15: TPS5600

41. Migden MR, Rischin D, Schmults CD, Guminski A, Hauschild A, Lewis KD et al. PD-1 blockade with cemiplimab in advanced cutaneous squamous-cell carcinoma. NEJM. 2018;379:341-51. 